\title{
Éditorial: Un nouveau siècle des lumières?
}

\author{
Nathalie Loye \\ Université de Montréal
}

"Je me dis: commençons par me faire un magasin d'idées, vraies ou fausses, mais nettes, en attendant que ma tête en soit assez fournie pour pouvoir les comparer et choisir. Cette méthode n'est pas sans inconvénients, je le sais, mais elle a réussi dans l'objet de m'instruire.»

(Jean-Jacques Rousseau, Confessions, Livre VI)

L'image proposée par Jean-Jacques Rousseau convient à ce que nous vous proposons dans ce numéro spécial : un « magasin d'idées ». Dans nos disciplines respectives, nous nous targuons d'être des spécialistes et de parfaitement connaître notre domaine d'expertise. Ainsi, nous nous savons aptes à trouver des solutions statistiques (ou autre) pour résoudre les problèmes que nous nous posons à nousmême dans nos programmes de recherche. Dans certains cas pourtant, tout près de nous, travaillent d'autres spécialistes dont les outils et les approches seraient une solution idéale à notre problème du moment, mais nous ne le savons simplement pas.

C'est autour de cette idée somme toute fort simple, qu'en juin 2010 nous avons réuni divers spécialistes des méthodes quantitatives en sciences humaines pour un colloque d'une journée intitulé Méthodes quantitatives et sciences humaines $2^{\text {ème }}$ édition. Pour être admis dans le cénacle, il fallait proposer une communication en lien avec l'utilisation de méthodes statistiques de pointe dans un quelconque domaine des sciences humaines. Furent abordés des thèmes aussi variés que l'imputation des données manquantes, les modèles multi-niveaux non paramétriques, divers problèmes liés aux modèles de mesure issus de la théorie de réponse à l'item (TRI), l'évaluation de l'invariance de la mesure ou encore l'étude des poussées de croissance ou des données longitudinales. Liés par leur intérêt (amour?) pour les statistiques, les participants à cette journée étaient des spécialistes en psychologie, en sexologie, en éducation ou encore en génie. Les échanges d'idées ont foisonné ce jourlà, ce numéro spécial en témoigne. Il présente un échantillon des sujets abordés en quatre articles et constitue notre « magasin d'idées ».

$\mathrm{Si}$ les savants du siècle des lumières lisaient notre numéro spécial, ils seraient fort impressionnés par l'utilisation des mathématiques et des statistiques que nous faisons au XXIème siècle. Condorcet notamment s'enthousiasmerait probablement devant les possibilités offertes par la simulation de données et les diverses estimations de l'habileté ou de la capacité d'un sujet avec un modèle 2PL de la théorie des items. Laurencelle et Germain nous font une très belle démonstration des forces et faiblesses des différents estimateurs basés sur des approches par maximum de vraisemblance, bayésienne ou utilisant les distributions a posteriori. Leurs conclusions permettraient sans aucun doute de raviver le débat entre Condorcet et d'Alembert autour de la pertinence d'utiliser des estimateurs biaisés. Pourtant Laurencelle et Germain offrent les arguments pour que Condorcet gagne la bataille.

L'une des armes en faveur de l'utilisation des statistiques en sciences humaine, au-delà des développements théoriques et des modèles qui ne cessent d'évoluer, est sans conteste la technologie. Elle a d'ailleurs permis à Laurencelle et Germain de simuler les données nécessaires à leur démonstration. Les avancées des outils informatiques nous amènent à l'heure actuelle à réaliser, parfois en quelques fractions de secondes, des calculs dans lesquels personne n'aurait même imaginé s'aventurer au siècle des lumières. La détection et la quantification des poussées de croissance est l'objet d'intérêt de Dandurand et Shultz. Ils nous présentent un nouvel outil sous la forme d'une interface graphique qui permet de détecter et de mesurer les minima et maxima locaux de données temporelles. Ce système automatisé est sans conteste un outil qui peut vraiment être 
utile, quel que soit le type de données temporelles, car il permet de les analyser rapidement, avec rigueur et de bénéficier d'une visualisation graphique des résultats.

Avec notre " magasin d'idées », nous souhaitons mettre en valeur la transversalité des méthodes statistiques. Harvey nous propose une belle illustration. Sur la base de modèles de Markov à états cachés, bien ancrés dans le domaine de la psychologie, il nous offre une démonstration de leur utilisation en éducation pour analyser des séquences de données répétées à travers le temps sur un même échantillon. Ces données lui permettent de développer et de valider un modèle général de développement des compétences pour interpréter les interactions entre superviseur et supervisé en situation de compagnonnage professionnel en soins infirmiers.
Enfin, Bourdeau, Sylvain et Delmas nous offrent un tutoriel pour appréhender les problèmes liés à la multicolinéarité des variables dans les modèles de régressions. La méthode est appliquée de manière didactique, assorties d'exercices, à des données issues d'un outil visant à mesurer l'adhésion à leur traitement de patients atteints du VIH. L'approche est elle aussi assurément transférable à d'autres types de données.

Maintenant que nous vous avons présenté notre « magasin d'idées », il ne nous reste qu'à vous souhaiter la bienvenue dans notre nouveau siècle des lumières et à vous souhaiter une bonne lecture.

Manuscript received 20 May 2011. 